Int. J. Theor. Phys., 1990, v. 29, N6, pp. 607-620.

\title{
Transitions: Contractions and Analytical Continuations of the Cayley-Klein Groups
}

N. A. Gromov

Department of Applied Mathematics, Komi Research Center of the USSR Academy of Sciences, Syktyvkar, 167610, USSR.

Received October 20, 1989

As a foundation for Klein's fundamental idea about the connection of geometry and its motion group and the unified description of all Cayley-Klein geometries, a method of group transitions including contractions as well as analytical continuations of the groups is developed. The generators and Casimir operators of an arbitrary Cayley-Klein group are obtained from those of the classical orthogonal group. The classification of all possible transitions between the Cayley-Klein groups is given. The physically important case of the kinematic groups is discussed.

\section{Introduction}

Sanjuan (1984) investigated the geometrical significance of the Inonu-Wigner contractions (Inonu and Wigner, 1953) and found that the very meaning of group contraction is deeply rooted in the relations of contrac-tions of the associated Cayley-Klein geometries. Lord (1985) related this idea to the projective characterization of the Cayley-Klein spaces in terms of absolutes. Pimenov (1965) gave a unified description of all $3^{n}$-dimensional CayleyKlein geometries and built the transformations of the elliptic geometry into arbitrary Cayley-Klein geometry. In accordance with the Erlangen Program due to F. Klein, each geometry is associated with a motion group. Then the transformations of the geometry induce the transfor-mations of the related motion group. We have used this idea to investigate the orthogonal (Gromov, 1978, 1981, 1982), unitary (Gromov, 1984), and symplectic (Gromov, 1985 ) groups from the viewpoint of transformations. It must be emphasized that the transformations under consideration in-clude contractions as well as analytical continuations of groups (when, for example, the Euclidean group transforms into the Poincare one). The present approach is based upon metrical concepts only and it is our hope that the method of transformations will be useful for physicists.

The organization of this paper is as follows: In Section 2 the three Cayley-Klein geometries on the line and their motion groups are regarded in detail. In Section 3 the motion groups of the nine Cayley-Klein geometries on the plane and their Casimir operators are studied. Section 4 is devoted 
to the generalization of the obtained results to higher dimensions. In Section 5 the interpretation of the four-dimensional Cayley-Klein spaces as the kinematics is given. The classification of the contractions and analytical continuations of the Cayley-Klein motion groups is regarded in Section 6; and finally in Section 7 the principal conclusions of this work are stated.

\section{The three fundamental geometries on a line}

We define the elliptic geometry on a line as follows. The circle $S_{1}^{*}=$ $\left\{\mathbf{x}^{\in} R_{2} \mid x_{0}^{* 2}+x_{1}^{* 2}=1\right\}$ on the Euclidean plane is invariant under rotations

$$
\begin{gathered}
x_{0}^{*^{\prime}}=x_{0}^{*} \cos \varphi^{*}-x_{1}^{*} \sin \varphi^{*} \\
x_{1}^{*^{\prime}}=x_{0}^{*} \sin \varphi^{*}-x_{1}^{*} \cos \varphi^{*}, \quad \varphi^{*} \in(-\pi, \pi)
\end{gathered}
$$

from the group $\mathrm{SO}_{2}$. We identify diametrically opposed points on the circle and introduce the intrinsic coordinate $\xi^{*}=x_{1}^{*} / x_{0}^{*}$ into it. Then the rotations (1) on the plane $R_{2}$ correspond to the translations on the line

$$
\xi^{*^{\prime}}=\frac{\xi^{*}-a^{*}}{1+\xi^{*} a^{*}}, \quad a^{*}=\operatorname{tg} \varphi^{*}
$$

where $\varphi^{*} \in(-\pi / 2, \pi / 2)$ and $a^{*} \in R$. The translations (2) form the motion group $G_{1}$ of the elliptic geometry on the line with the composition law

$$
a^{*^{\prime}}=\frac{a^{*}+a_{1}^{*}}{1-a^{*} a_{1}^{*}}
$$

For the representation $T\left(g\left(\varphi^{*}\right)\right) f\left(\mathbf{x}^{*}\right)=f\left(g^{-1}\left(\varphi^{*}\right) \mathbf{x}^{*}\right)$ of the group $S_{2}$ in the space of differentiable functions on $\mathbf{R}_{2}$ the generator

$$
X^{*} f\left(\mathbf{x}^{*}\right)=\left.\frac{d}{a \varphi^{*}}\left[T\left(g\left(\varphi^{*}\right)\right) f\left(\mathbf{x}^{*}\right)\right]\right|_{\varphi^{*}=0}
$$

corresponding to the rotation (1) assumes the form

$$
X^{*}\left(x_{0}^{*}, x_{1}^{*}\right)=x_{1}^{*} \frac{\partial}{\partial x_{0}^{*}}-x_{0}^{*} \frac{\partial}{\partial x_{1}^{*}}
$$

It is easy to obtain the generator $Z^{*}\left(\xi^{*}\right)$ of the representation of the group $G_{1}$ in the space of differentiable functions on the line

$$
Z^{*}\left(\xi^{*}\right)=\left(l+\xi^{* 2}\right) \frac{\partial}{\partial \xi^{*}}
$$

For completeness we give the form of the generator of the rotation (1),

$$
X^{*}=\left(\begin{array}{cc}
0 & -1 \\
1 & 0
\end{array}\right)
$$

We define now the fundamental map

$$
\begin{gathered}
\psi: R_{2} \rightarrow R_{2}\left(j_{1}\right) \\
\psi x_{0}^{*}=x_{0}, \quad \psi x_{1}^{*}=j_{1} x_{1}
\end{gathered}
$$


where Cartesian coordinates $x_{0}^{*}, x_{1}^{*} \in R_{2}, x_{0}, x_{1} \in R_{2}\left(j_{1}\right)$, the parameter $j_{1}$ may be equal to the real unit 1 or to the Clifford dual unit $\iota_{1}$ or to the imaginary unit $i$. The dual unit is not equal to zero, but its square is $\iota_{1}^{2}=0$. Division of a real or complex number by the dual unit is not defined. We assume that the division of a dual unit by itself is equal to the real unit, $\iota_{1} / \iota_{2}=1$. The last property is equivalent to the fact that the equation $a \iota_{1}=b \iota_{1}$ has only one solution $a=b$ in the real or complex number field.

The fundamental map $\psi$ transforms the Euclidean plane $R_{2}$ into the plane $R_{2}\left(j_{1}\right)$, the geometry of which is defined by the metric $\mathbf{x}^{2}\left(j_{1}\right)=x_{0}^{2}+$ $j_{1}^{2} x_{1}^{2}$. Then, as is well known, $R_{2}(i)$ is the Minkowskian plane, $R_{2}\left(\iota_{1}\right)$ is the Galilean plane, and $R_{2}(l)=R_{2}$ is the Euclidean plane.

Our main idea is that the fundamental map $\psi$ of geometries induces the transformations of associated groups of motions and their algebras. We now show how to obtain the transformations of motions and generators.

The measure of angles on the Euclidean plane $R_{2}$ is defined by the ratio $x_{1}^{*} / x_{0}^{*}$, which goes to $j_{1} x_{1} / x_{0}$ under the map (7). Then the angle transforms as follows: $\psi \varphi^{*}=j_{1} \varphi$. Replacing in (1) the coordinates and the angle according to the map and multiplying the second equation by the parameter $j_{1}^{-1}$, we obtain the rotations on the plane $R_{2}\left(j_{1}\right)$,

$$
\begin{gathered}
x_{0}^{\prime}=x_{0} \cos j_{1} \varphi-x_{1} j_{1} \sin j_{1} \varphi \\
x_{1}^{\prime}=x_{0} j_{1}^{-1} \sin j_{1} \varphi+x_{1} \cos j_{1} \varphi
\end{gathered}
$$

The function of the dual argument is determined by its Taylor series expansion. Specifically, we have $\cos \iota_{1} \varphi=1, \sin \iota_{1} \varphi=\iota_{1} \varphi$. The rotations (8) form a one-parameter group, which will be denoted by the symbol $S O_{2}\left(j_{1}\right)$. The elements of the group $\mathrm{SO}_{2}\left(\iota_{1}\right)$ are the Galilean transformations and the elements of the $S_{2}(i)$ are the Lorentz transformations if $x_{0}$ is regarded as a temporal axis and $x_{1}$ as a spatial one. The group parameters $\varphi$ belong to the domains $\Phi\left(j_{1}\right)$, where $\Phi(1)-(-\pi / 2, \pi / 2), \Phi\left(\iota_{1}\right)=\Phi(i)=R$.

The circles

$$
S_{1}\left(j_{1}\right)=\left\{\mathbf{x} \in R_{2}\left(j_{1}\right) \mid x_{0}^{2}+j_{1}^{2} x_{1}^{2}=1\right\}
$$

on the planes $R_{2}\left(j_{1}\right)$ (Figure 1 ) are invariant under the rotations (8). Identification of the diametrically opposed points on the circles gives the upper semicircle for $j_{1}=1$ on the upper simply connected part of the circles for $j_{1}=\iota_{1}, i$. The intrinsic coordinate $\xi^{*}$ transforms as follows: $\psi \xi^{*}=j_{1} \xi$. Then for the translations on the line we have

$$
\xi^{\prime}=\frac{\xi-a}{1+j_{1}^{2} \xi a}, \quad a=j_{1}^{-1} t g j_{1} \varphi
$$

where $a \in R$. The transformations (9) generate the one-parameter groups of motion $G_{1}\left(j_{1}\right)$ on the elliptic line $\left(j_{1}=1\right)$, on the parabolic line $\left(j_{1}=\iota_{1}\right)$, or on the hyperbolic line $\left(j_{1}=i\right)$.

In the space of differentiable functions on the $R_{2}\left(j_{1}\right)$ the generator $X(\mathbf{x})$ of the representation of the group $S_{2}\left(j_{1}\right)$ is defined as

$$
X f(\mathbf{x})=\left.\frac{d}{d \varphi}[T(g(\varphi)) f(\mathbf{x})]\right|_{\varphi=0}
$$

Under the map $\psi$, the derivative $d / d \varphi^{*}$ goes into $j_{1}^{-1} d / d \varphi$; therefore, we need to multiply the generator $X^{*}\left(\psi \mathbf{x}^{*}\right)$ by the parameter $j_{1}$ in order to 
have the generator $X(\mathbf{x})$. The law of transformation of the generator takes the form

$$
X(\mathbf{x})=j_{1} X^{*}\left(\psi \mathbf{x}^{*} 0=j_{1}^{2} x_{1} \frac{\partial}{\partial x_{0}}-x_{0} \frac{\partial}{\partial x_{1}}\right.
$$

The same is true for the generator $Z$,

$$
Z(\xi)=j_{1} Z^{*}\left(\psi \xi^{*}\right)=\left(1+j_{1}^{2} \xi^{2}\right) \frac{\partial}{\partial \xi}
$$

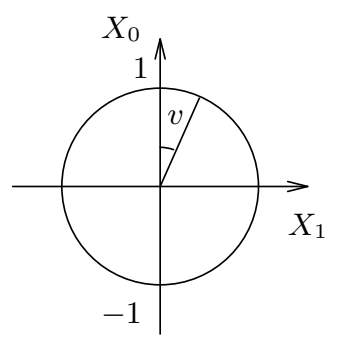

a) $j_{1}=1$

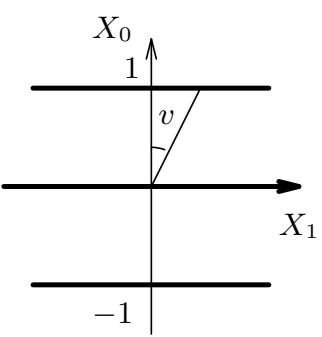

b) $j_{1}=\iota_{1}$

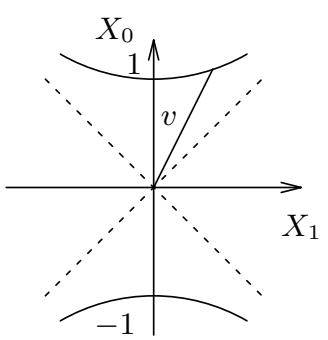

a) $j_{1}=i$

Fig.1. The circles $S_{1}\left(j_{1}\right)$ on the planes $R_{2}\left(j_{1}\right)$.

$$
\text { (a) } j_{1}=1,(b) j_{1}=\iota_{1},(c) j_{1}=i
$$

It is easy to determine the law of transformation of the matrix generator (6): the nonzero matrix element -1 above the principal diagonal is replaced by $-j_{1}^{2}$ and all the others are not changed, i.e.,

$$
X^{*}=\left(\begin{array}{cc}
0 & -j_{1}^{2} \\
1 & 0
\end{array}\right)
$$

The groups $G_{1}\left(j_{1}\right)$ and $S O_{2}\left(j_{1}\right)$ are very similar (their Lie algebras are isomorphic). Hereafter we shall understand under the Cayley-Klein groups the groups $G_{1}\left(j_{1}\right)$ as well as $S O_{2}\left(j_{1}\right)$ and under the Cayley-Klein spaces the spaces $S_{1}\left(j_{1}\right)$ as well as $R_{2}\left(j_{1}\right)$. The same rule will be observed for higher dimensions.

We have regarded in detail the simplest groups $S O_{2}\left(j_{1}\right), G_{1}\left(j_{1}\right)$ in order to demonstrate the principal ideas of the method of transformations when they are not camouflaged by the mathematical calculations. The main stages are as follows: (i) one defines the map $\psi$ from the Euclidean space to the arbitrary Cayley-Klein space; (ii) one finds the transformation laws for the motions, generators, etc., of a group; and (iii) one determines the motions, generators, etc., of the Cayley-Klein group from the appropriate quantities of the classical orthogonal group with the help of (ii). The simple method of transformations enables us to describe all Cayley-Klein groups knowing only the classical orthogonal group.

At first sight there are two different processes in a group theory. One is a group contraction as defined by Inonu and Wigner (1953), and the other 
is an analytical continuation of a group. The last is used, for example, in field theory when imaginary time is regarded. From the viewpoint of transformation, both processes have a common nature, namely the replacement of real group parameters by dual ones (contraction) or by imaginary ones (continuation) that are the continuation from the real number field into the dual or complex ones.

\section{The nine Cayley-Klein groups}

The map

$$
\begin{gathered}
\psi: R_{3} \rightarrow R_{3}(\mathbf{j}) \\
\psi x_{0}^{*}=x_{0}, \quad \psi x_{1}^{*}=j_{1} x_{1}, \quad \psi x_{2}^{*}=j_{1} j_{2} x_{2}
\end{gathered}
$$

where $\mathbf{j}=\left(j_{1}, j_{2}\right) ; j_{1}=1, \iota, i ; j_{2}=1, \iota_{2}, i$; the $\iota_{1}$ and $\iota_{2}$ are two different dual units with the properties , $\iota_{1}^{2}=0, \iota_{2}^{2}=0$ but $\iota_{1} \iota_{2}=\iota_{2} \iota_{1} \neq 0$ and $\iota_{k} / \iota_{k}=1, k=1,2$, (but not $\iota_{1} / \iota_{2}$ or $\iota_{2} / \iota_{1}$; these constructions are not defined) transforms the three-dimensional Euclidean space into the spaces $R_{3}(\mathbf{j})$. The nine Cayley-Klein plane geometries are realized on the spheres

$$
S_{2}(\mathbf{j})=\left\{\mathbf{x} \in R_{3}\left(\mathbf{j} \mid x_{0}^{2}+j_{1}^{2} x_{1}^{2}+j_{1}^{2} j_{2}^{2} x_{2}^{2}=1\right\}\right.
$$

in these spaces. Table I gives the identification of the Cayley-Klein geometries with the parameters $\mathbf{j}$.

The angle $\varphi_{\mu \nu}^{*}$ in the coordinate plane $\left\{x_{\mu}^{*}, x_{\nu}^{*}\right\}, \mu<\nu, \mu, \nu=0,1,2$, of the space $R_{3}$ is determined by the ratio $x_{\nu}^{*} / x_{\mu}^{*}$ and transforms under the map (13) as $\psi \varphi_{\mu \nu}^{*}=\left(\prod_{m=\mu+1}^{\nu} j_{m}\right) \varphi_{\mu \nu}$. Then, as in the preceding section, we obtain the rotations in the plane $\left\{x_{\mu}, x_{\nu}\right\}$ of the space $R_{3}(\mathbf{j})$ in the form

$$
\begin{gathered}
x_{\mu}^{\prime}=x_{m} u \cos \left(\varphi_{\mu \nu} \prod_{m=\mu+1}^{\nu} j_{m}\right)-x_{n} u\left(\prod_{m=\mu+1}^{\nu} j_{m}\right) \sin \left(\varphi_{\mu \nu} \prod_{m=\mu+1}^{\nu} j_{m}\right) \\
x_{\nu}^{\prime}=x_{m} u\left(\prod_{m=\mu+1}^{\nu} j_{m}\right) \sin \left(\varphi_{\mu \nu} \prod_{m=\mu+1}^{\nu} j_{m}\right)
\end{gathered}
$$

The matrix generators of rotations are

$$
X_{01}=\left(\begin{array}{ccc}
0 & -j_{1}^{2} & 0 \\
1 & 0 & 0 \\
0 & 0 & 0
\end{array}\right), \quad X_{02}=\left(\begin{array}{ccc}
0 & 0 & -j_{1}^{2} j_{2}^{2} \\
0 & 0 & 0 \\
1 & 0 & 0
\end{array}\right), \quad X_{12}=\left(\begin{array}{ccc}
0 & 0 & 0 \\
0 & 0 & -j_{2}^{2} \\
0 & 1 & 0
\end{array}\right)
$$

They form the basis of the Lie algebra of the group $\mathrm{SO}_{3}(\mathbf{j})$.

The transformation laws of the generators of the representation of the group $S_{3}(\mathbf{j})$ in the space of differentiable functions on the $R_{3}(\mathbf{j})$ are the same as those of the angles, namely

$$
X_{\mu \nu}(\mathbf{x})=\left(\prod_{m=\mu+1}^{\nu} j_{m}\right) X_{\mu \nu}^{*}\left(\psi \mathbf{x}^{*}\right)
$$

and the qenerators are

$$
X_{\mu \nu}(\mathbf{x})=\left(\prod_{m=\mu+1}^{\nu} j_{m}^{2}\right) x_{\nu} \frac{\partial}{\partial x_{\mu}}-x_{\mu} \frac{\partial}{\partial x_{\nu}}
$$


Knowing the generators, it is easy to get the Lie brackets of the Lie algebra of the motion group. But we obtain the Lie brackets from those of

Table I. The Nine Cayley-Klein Geometries

\begin{tabular}{cccc}
\hline & $j_{1}=1$ & $j_{1}=\iota_{1}$ & $j_{1}=i$ \\
\hline$j_{1}=1$ & Elliptic & Euclidean & $\begin{array}{c}\text { Lobachevskian } \\
\text { (hyperbolic) }\end{array}$ \\
$j_{2}=\iota_{2}$ & Semielliptic & Galilean & $\begin{array}{c}\text { Semihyperbolic } \\
\text { (co-Minkowskian) }\end{array}$ \\
$j_{2}=i$ & Anti-de Sitter & Minkowskian & De Sitter \\
\hline
\end{tabular}

the orthogonal group. We introduce new designations for the generators: $X_{01}^{*}=H^{*}, X_{02}^{*}=P^{*}, X_{12}^{*}=K^{*}$. As is well known, the Lie brackets of the group $\mathrm{SO}_{3}$ are

$$
\left[H^{*}, P^{*}\right]=K^{*}, \quad\left[P^{*}, K^{*}\right]=H^{*}, \quad\left[H^{*}, K^{*}\right]=-P^{*}
$$

The generators of the Lie algebra of the group $\mathrm{SO}_{3}$ are transformed as follows: $H=j_{1} H^{*}, P=j_{1} j_{2} P^{*}, K=j_{2} K^{*}$. Then $h^{*}=j_{1}^{-1} H, p^{*}=$ $j_{1}^{-1} j_{2}^{-1} P, K^{*}=j_{2}^{-1} K$. Substituting these formulas for the Lie brackets (18) and multi-plying each Lie bracket by its denominator, we obtain the Lie brackets of the groups $\mathrm{SO}_{3}(j)$ :

$$
[H, P]=j_{1}^{2} K, \quad[P, K]=j_{2}^{2} H, \quad[H, K]=-P
$$

The Cayley-Klein spaces $S_{2}(\mathbf{j})$ for $j_{1}=1, \iota_{1}, i$ and $j_{2}=\iota_{2}, i$ may be regarded as kinematics, in which case the intrinsic coordinate $\xi_{1}=x_{1} / x_{0}$ is interpreted as a temporal axis and $\xi_{2}=x_{2} / x_{0}$ as a spatial one. Then the generators of the groups $\mathrm{SO}_{3}(\mathbf{j})$ may be interpreted as follows: $H$ is the generator of the time translation, $P$ is the generator of the space translation, and $K$ is the generator of the Galilean transformation for $j_{2}=\iota_{2}$ or of the Lorentz transformation for $j_{2}=i$.

The requirement that the final formulas should not contain undefined expressions like $\iota^{-1}$ suggests a way to find the transformation laws of the algebraic constructions. Let an algebraic quantity $Q^{*}$ be some function of algebraic quantities $A_{1}^{*}, \ldots, A_{k}^{*}$, i.e., $Q^{*}=Q^{*}\left(A_{1}^{*}, \ldots, A_{k}^{*}\right)$. Let the transformation laws of $A_{1}^{*}, \ldots, A_{k}^{*}$ be known, $A_{1}=a_{1} A_{1}^{*}, \ldots, A_{k}=a_{k} A_{k}^{*}$, where the coefficients $a_{1}, \ldots, a_{k}$ are products of the parameters $\mathbf{j}$. Substituting $A_{1}^{*}=a_{1}^{-1} A_{1}, \ldots, A_{k}^{*}=a_{k}^{-1} A_{k}$ for the function $Q^{*}$, we obtain the function $Q^{*}\left(a_{1}^{-1} A_{1}, \ldots, a_{k}^{-1} A_{k}\right)$, which contains, generally speaking, undefined quantities when the parameters $\mathbf{j}$ are dual. Therefore, the last function must be multiplied by a coefficient $q$ such that the final function is well defined. The transformation law of $Q$ is then

$$
Q\left(A_{1}, \ldots, A_{k}\right)=q Q^{*}\left(a_{1}^{-1} A_{1}, \ldots, a_{k}^{-1} A_{k}\right)
$$

We demonstrate this with the help of the Casimir operator. The only Casimir operator of the group $\mathrm{SO}_{3}$ is given by

$$
C_{2}^{*}\left(H^{*}, P^{*}, K^{*}\right)=H^{* 2}+P^{* 2}+K^{* 2}
$$

Substituting $H^{*}=j_{1}^{-1} H, P^{*}=j_{1}^{-1} j_{2}^{-1} P, K^{*}=j_{2}^{-1} K$ in the formula (21), we obtain

$$
C_{2}^{*}\left(j_{1}^{-1} H, \ldots\right)=j_{1}^{-2} H^{2}+j_{1}^{-2} P^{2}+j_{2}^{-2} K^{2}
$$


When $j_{1}=\iota, j_{2}=\iota_{2}$ the most singular coefficient in (22) $j_{1}^{-2} j_{2}^{-2}$. Multiplying both parts of equation (22) by $j_{1}^{2} j_{2}^{2}$, we are safe from undefined quantities.

Then the transformation law and the formula for the Casimir operator of the group $\mathrm{SO}_{3}(\mathbf{j})$ are in the form

$$
C_{2}(H, \ldots ; \mathbf{j})=j_{1}^{2} j_{2}^{2} C_{2}^{*}\left(j_{1}^{-1} H, \ldots\right)=j_{2}^{2} H^{2}+P^{2}+j_{1}^{2} K^{2}
$$

The Casimir operator of the two-dimensional Galilean group $\mathrm{SO}_{3}\left(\iota_{1}, \iota_{2}\right)$ is well known to be $C_{2}\left(\iota_{1}, \iota_{2}\right)=P_{2}$ (see Levy-Leblond, 1971); for the two-dimensional Poincare group $S_{3}\left(\iota_{1}, i\right)$ it is given by the expression $C_{2}\left(\iota_{1}, i\right)=P^{2}-H^{2}$; and the Casimir operator of the group $S_{3}\left(\iota_{1}, 1\right)=$ $S O(2,1)$ is equal to $C_{2}(i, 1)=H^{2}+P^{2}-K^{2}$ (Mukunda, 1967). All these Casimir operators are given by equation (23) under the appropriate values of parameters $\mathbf{j}$.

\section{Generalization to higher demensions}

The $n$-dimensional Cayley-Klein geometries are realized on the spheres

$$
S_{n}(\mathbf{j})=\left\{x \in R_{n+1}(\mathbf{j}) \mid x_{0}^{2}+\sum_{m=1}^{n}\left(\prod_{m=1}^{k} j_{m}^{2}\right) x_{k}^{2}=1\right\}
$$

in the spaces $R_{n+1}(\mathbf{j})$ which are obtained from the Euclidean space $R_{n+1}$ by the map

$$
\begin{gathered}
\psi: R_{n+1} \rightarrow R_{n+1}(\mathbf{j}) \\
\psi x_{0}^{*}=x_{0}, \quad \psi x_{k}^{*}=\left(\prod_{m=1}^{k} j_{m}\right) x_{k}
\end{gathered}
$$

where $\mathbf{j}=\left(j_{1}, \ldots, j_{n}\right) ; j_{k}=1, \iota_{k}, i ; \iota_{k} \iota_{r}=\iota_{r} \iota_{k} \neq 0$ for $k \neq r$ but $\iota_{k}^{2}=0, k=$ $1,2, \ldots, n$.

The rotations in the planes $\left\{x_{\mu}, x_{\nu}\right\}$, the transformation laws of the generators, and the formulas for these generators are given by (14), (16), and (17), respectively, but $\mu, \nu=0,1, \ldots, n, \mu<\nu$. The nonzero matrix elements of the rotation generators are

$$
\left(X_{\mu \nu}\right)_{\nu \mu}=1, \quad\left(X_{\mu \nu}\right)_{\mu \nu}=-\left(\prod_{m=\mu+1}^{\nu} j_{m}^{2}\right)
$$

The Lie brackets (or commutators) of the group $S O_{n+1}(\mathbf{j})$ are most easily constructed from those of $S O_{n+1}$, as was done in Section 3. The nonzero commutators are

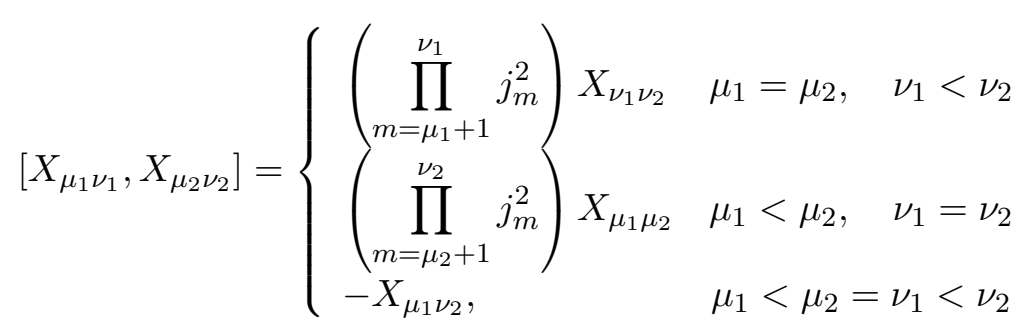


As is well known, there are $[(n+1) / 2]$ independent Casimir operators of the group $S O_{n+1}$, where $[x]$ is the integral part of the number $x$. For even $n=2 k$, the Casimir operators are given by (Barut and Raczka, 1977)

$$
C_{2 p}^{*}\left(X_{\mu \nu}^{*}\right)=\sum_{\alpha_{1}, \ldots, \alpha_{2 p}=0}^{n} X_{\alpha_{1} \alpha_{2}}^{*} X_{\alpha_{2} \alpha_{3}}^{*} \ldots X_{\alpha_{2 p} \alpha_{1}}^{*}
$$

where $p=1,2, \ldots, k$. The operator

$$
C^{* 1 / 2}\left(X_{\mu \nu}^{*}\right)=\sum_{\alpha_{1}, \ldots, \alpha_{n+1}=0}^{n} \varepsilon_{\alpha_{1} \ldots \alpha_{n+1}} X_{\alpha_{1} \alpha_{2}}^{*} X_{\alpha_{3} \alpha_{4}}^{*} \ldots X_{\alpha_{n} \alpha_{n+1}}^{*}
$$

is added to the operators (27) for odd $n=2 k+1$, where $\varepsilon_{\alpha_{1} \ldots \alpha_{n+1}}$ is the antisymmetric unit tensor.

Another definition of the Casimir operators was given by Gel'fand (1950). By his definition the Casimir operator $C_{2 p}^{*^{\prime}}$ is the sum of all $2 p$-dimensional principal minors of the antisymmetric matrix $A$ which is constructed from the generators $X_{\mu \nu}$, namely $(A)_{\mu \nu}=X_{\mu \nu}^{*},(A)_{\nu \mu}=-X_{\mu \nu}^{*}$.

We get the Casimir operators of $S O_{n+1}(\mathbf{j})$ in a quite similar manner as in Section 3. From (16) we have $X_{\mu \nu}^{*}=\left(\prod_{m=\mu+1}^{\nu} j_{m}^{-1}\right) x_{\mu \nu}$ and substitute in (27). The addend $X_{0 n} X_{n 0} \ldots X_{n 0}$ has the most singular coefficient $\prod_{m=1}^{n} j_{m}^{-2 p}$. Then the transformation laws of the Casimir operators $C_{2 p}$ are in the form

$$
C_{2 p}\left(\mathbf{j} ; X_{\mu \nu}\right)=\left(\prod_{m=1}^{n} j_{m}^{2 p}\right) C_{2 p}^{*}\left(X_{\mu \nu} \prod_{m=\mu+1}^{\nu} j_{m}^{-1}\right)
$$

and the formulas for the operators themselves are

$$
\begin{aligned}
C_{2 p}(\mathbf{j})= & \sum_{\alpha_{1}, \ldots, \alpha_{2 p}=0}^{n}\left(\prod_{m=1}^{n} j_{m}^{2 p}\right) \prod_{r=1}^{2 p}\left(\prod_{l_{r}=\mu_{r}+1}^{\nu_{r}} j_{l_{r}}^{-1}\right) \\
& \times X_{\alpha_{1} \alpha_{2}} X_{\alpha_{2} \alpha_{3}} \ldots X_{\alpha_{2 p} \alpha_{1}}
\end{aligned}
$$

where $\mu_{r}=\min \left(\alpha_{r}, \alpha_{r+1}\right), \nu_{r}=\max \left(\alpha_{r}, \alpha_{r+1}\right), r=1,2, \ldots, 2 p-1, \mu_{2 p}=$ $\min \left(\alpha_{1}, \alpha_{2 p}\right)$, and $\nu_{2 p}=\max \left(\alpha_{1}, \alpha_{2 p}\right.$.

The Casimir operators $C_{2 p}^{*^{\prime}}$ and $C^{* 1 / 2}$ after replacing the generators according to (16) must be multiplied by the least common denominators of the coefficients of the addend in the sums. These least common denominators are easily found with the help of mathematical induction. We give the final form for the transformation laws of Casimir operators:

$$
\begin{gathered}
C_{2 p}^{\prime}\left(\mathbf{j} ; X_{\mu \nu}\right)=\left(\prod_{m=1}^{p-1} j_{m}^{2 m} j_{n-m+1}^{2 m} \prod_{l=p}^{n-p+1} j_{l}^{2 p}\right) C_{2 p}^{*^{\prime}}\left(X_{\mu \nu} \prod_{l=\mu+1}^{\nu} j_{l}^{-1}\right) \\
C^{1 / 2}\left(\mathbf{j} ; X_{\mu \nu}\right)=\left(j_{(n+1) / 2}^{(n+1) / 2} \prod_{m=1}^{(n-1) / 1} j_{m}^{m} j_{n-m+1}^{m}\right) \\
\times C^{* 1 / 2}\left(X_{\mu \nu} \prod_{l=\mu+1}^{\nu} j_{l}^{-1}\right)
\end{gathered}
$$


The operator $C_{2 p}(\mathbf{j})$ [or $\left.C^{1 / 2}(\mathbf{j})\right]$ commutes with all generators $X_{\mu \nu}$ of the group $S O_{n+1}(\mathbf{j})$. Indeed, when we calculate the zero equal commutator $\left[C_{2 p}^{*}, X_{\mu \nu}^{*}\right]$ we have the addends of the same form but with a different sign (plus or minus). The sum of these addends is equal to zero. Under the transformations (16) and (29), both these addends are multiplied by the same combination of parameters with even-power products of $j_{k}$. Therefore both addends either simultaneously change sign or are equal to zero or do not change, but in any case their sum is equal to zero. Besides the operators $C_{2 p}(\mathbf{j})$ for $p=1,2, \ldots,[(n+1) / 2]$ are linearly independent because they are formed by generators $X_{\mu \nu}$ of different degree. Using the method of Chaichian et al. (1983), we can prove that for any $\mathbf{j}$ the number of Casimir operators of $S O_{n+1}(\mathbf{j})$ is equal to $[(n+1) / 2]$. Thus, we have constructed all Casimir operators of the group $S O_{n+1}(\mathbf{j})$ for any $\mathbf{j}$ parameters.

\section{$5 \quad$ Kinematics as the Cayley-Klein spaces}

Starting from very general physical principles, Bacry and Levy-Leblond (1968) classified all possible kinematics. Almost all kinematics are fourdimensional Cayley- Klein spaces $S_{4}(\mathbf{j})$ with the appropriate physical interpretation of the intrinsic coordinates as spatial and temporal ones. There are two possibilities (Gromov, 1986).

First we can regard the coordinate $\xi_{1}=x_{1} / x_{0}=t$ as the temporal axis and the coordinates $\xi_{k+1}=x_{k+1} / x_{0}-r_{k}, k=1,2,3$, as the spatial axes. In this case the physical postulate of isotropic space restricts the parameters $j_{3}, j_{4}$ as follows: $j_{3}=j_{4}=1$ and the postulate "inertial transformations (or boosts) in any given direction form a noncompact subgroup" restricts the parameters $j_{2}$, namely $j_{2}=\iota_{2}, i$ (see Figure 1 ). Table I gives the identification of the Cayley-Klein spaces $S_{4}\left(j_{1}, j_{2}, 1,1\right)=S_{4}\left(j_{1}, j_{2}\right)$ with six kinematics.

The generators of the group $S O_{5}\left(j_{1}, j_{2}, 1,1\right)=S O_{5}\left(j_{1}, j_{2}\right)$ are physically interpreted as follows: $X_{01}=H$ is the generator of time translation; $X_{0, k+1}=P_{k}, k=1,2,3$ are the generators of space translations; $X_{1, k+1}=K_{k}$ are the boost generators; and $X_{34}=J_{1}, X_{24}=-J_{2}$, and $X_{23}=J_{3}$ are the generators of spatial rotations. The Lie brackets of these generators are

$$
\begin{gathered}
{[\mathbf{J}, \mathbf{J}]=\mathbf{J}, \quad[\mathbf{J}, \mathbf{P}]=\mathbf{P}, \quad[\mathbf{J}, \mathbf{K}]=\mathbf{K}} \\
{[H, \mathbf{J}]=0, \quad[H, \mathbf{K}]=\mathbf{P}, \quad[H, \mathbf{P}]=-j_{1}^{2} \mathbf{K}} \\
{[\mathbf{P}, \mathbf{P}]=j_{1}^{2} j_{2}^{2} \mathbf{J}, \quad[\mathbf{K}, \mathbf{K}]=j_{2}^{2} \mathbf{J}, \quad\left[P_{k}, K_{l}\right]=-j_{\delta_{k l}}^{2} H}
\end{gathered}
$$

where the notation $[\mathbf{X}, \mathbf{Y}]=\mathbf{Z}$ is shorthand for $\left[X_{k}, Y_{l}\right]=\varepsilon_{k l m} Z_{m}$.

There are two independent Casimir operators of the group $\mathrm{SO}_{5}$,

$$
\begin{gathered}
C_{2}^{*^{\prime}}=H^{* 2}+\mathbf{P}^{* 2}+\mathbf{K}^{* 2}+\mathbf{J}^{* 2} \\
X_{4}^{*^{\prime}}=\left(H^{*} \mathbf{J}^{*}-\mathbf{P}^{*} \times \mathbf{K}\right)^{2}+\left(\mathbf{P}^{*}, \mathbf{J}^{*}\right)^{2}+\left(\mathbf{K}^{*}, \mathbf{J}^{*}\right)^{2}
\end{gathered}
$$

Taking into account the transformation laws of the generators of the group $S O_{5}\left(j_{1}, j_{2}\right), H-j_{1} H^{*}, \mathbf{P}=j_{1} j_{2} \mathbf{P}^{*}, \mathbf{K}=j_{2} \mathbf{K}^{*}$, , and $\mathbf{J}=\mathbf{J}^{*}$, we obtain from equation (31) the Casimir operators of $S_{5}\left(j_{1}, j_{2}\right)$ in the form

$$
\begin{gathered}
C_{2}^{\prime}\left(j_{1}, j_{2}\right)=j_{2}^{2} H^{2}+\mathbf{P}^{2}+j_{1}^{2} \mathbf{K}^{2}+h_{1}^{2} j_{2}^{2} \mathbf{J}^{2} \\
C_{4}^{\prime}\left(j_{1}, j_{2}\right)=\left(j_{2}^{2} H \mathbf{J}-\mathbf{P} \times \mathbf{K}\right)+j_{2}^{2}(\mathbf{P}, \mathbf{J})^{2}+j_{1}^{2} j_{2}^{2}(\mathbf{K}, \mathbf{J})^{2}
\end{gathered}
$$


For $j_{1}=j_{2}=i$ the formulas (35) give the Casimir operators of the de Sitter group, and for $j_{1}=1$ and $j_{2}=i$ they give those of the Poincare group (compare with Barut and Raczka, 1977). For $j_{1}=1, i$ and $j_{2}=\iota_{2}$ we have the Casimir operators of the semielliptic and semihyperbolic groups (or the Newton-Hooke groups) (compare with Derom and Dubois, 1972). For $j_{1}=\iota_{1}$ and $j_{2}=\iota_{2}$ we obtain from (35) the Casimir operators of the Galilean group (compare with Levy-Leblond, 1971).

The second way is to interpret the geometrical coordinate $\xi_{4}=x_{4} / x_{0}$ as the temporal axis and the coordinates $\xi_{k}=x_{k} / x_{0}, k=1,2,3$, as the spatial axes. Then the physical interpretation of the generators of the group $S O_{5}\left(j_{1}, 1,1, \iota_{4}\right)$ follows from their notation, namely $X_{04}=H, x_{0 k}=$ $-P_{k}, X_{k 4}=K_{k}, k=1,2,3, X_{23}=J_{1}, X_{13}=-J_{2}, X_{12}=J_{3}$. We may write the Lie brackets of these generators

$$
\begin{array}{cll}
{[\mathbf{J}, \mathbf{J}]=\mathbf{J},} & {[\mathbf{J}, \mathbf{P}]=\mathbf{P},} & {[\mathbf{J}, \mathbf{K}]=\mathbf{K}} \\
{[H, \mathbf{J}]=0,} & {[H, \mathbf{K}]=0,} & {[H, \mathbf{P}]=j_{1}^{2} \mathbf{K}} \\
{[\mathbf{P}, \mathbf{P}]=j_{1}^{2} \mathbf{J},} & {[\mathbf{K}, \mathbf{K}]=0,} & {\left[P_{k}, K_{l}\right]=\delta_{k l} H}
\end{array}
$$

Comparing (36) with the Lie brackets of Bacry and Levy-Leblond (1968), we find that the group $S O_{5}\left(\iota_{1}, 1,1, \iota_{4}\right)$ is identical with the Carroll kinematic group first described by Levy-Leblond (1965), the group $S O_{5}\left(1,1,1, \iota_{4}\right)$ with the kinematic group $I S O(4)$ (or $P_{+}^{\prime}$ ), and $S O_{5}\left(i, 1,1, \iota_{4}\right)$ with the paraPoincare group $P_{-}^{\prime}$. Further taking into account that the parameter $j_{1}$, defines the sign of constant curvature of Cayley-Klein spaces, we conclude that the group $\mathrm{SO}_{5}\left(1,1,1, \iota_{4}\right)$ is the motion group of the Carroll kinematic with positive curvature, and the group $S_{5}\left(i, 1,1, \iota_{4}\right)$ is the motion group of the Carroll kinematic with negative curvature. The Casimir operators of the Carroll kinematic groups are obtained from (34) by renotation of the generators according to the new interpretation and with the help of the transformation laws (31). They are written in the form

$$
\begin{gathered}
C_{2}^{\prime}\left(j_{1}\right)=H^{2}+j_{1}^{2} \mathbf{K}^{2} \\
C_{4}^{\prime}\left(j_{1}\right)=(H \mathbf{J}-\mathbf{P} \times \mathbf{K})^{2}+j_{2}^{2}(\mathbf{K}, \mathbf{J})^{2}
\end{gathered}
$$

For $j_{1}=\iota_{2}$, the Casimir operators (37) coincide with those of the Carroll group found by Levy-Leblond (1965).

Bacry and Levy-Leblond have described 11 kinematic groups. Nine of them are geometrically interpreted as the Cayley-Klein spaces. The kinematic group "para-Galilei" $G^{\prime}$ is obtained from the Galilei group $S_{5}\left(\iota_{1}, \iota_{2}, 1,1\right)$ by the exchange $\mathbf{P} \rightarrow \mathbf{K}, \mathbf{K} \rightarrow \mathbf{P}$, that is, by the new interpreta-tion of generators when the space translation generators of the Galilei kinematic are regarded as the boost generators of the "para-Galilei" kine-matic and vice versa. The static kinematic is not identified with any Cayley-Klein space.

\section{The classification of the transitions}

Up to this point we have regarded the transition from the Euclidean geometry to arbitrary Cayley-Klein geometry and the induced transition from the classical orthogonal group to the motion group of arbitrary Cayley-Klein space. It is useful to investigate also the transitions between all CayleyKlein geometries. 
Let us (formally) define the map

$$
\begin{gathered}
\omega: R_{n+1}(\mathbf{j}) \rightarrow R_{n+1}\left(\mathbf{j}^{\prime}\right) \\
\omega x_{0}=x_{0}^{\prime}, \quad \omega x_{k}=x_{k}^{\prime} \prod_{m=1}^{k} j_{m}^{\prime} j_{m}^{-1}, \quad k=1,2, \ldots, n
\end{gathered}
$$

This map induces transformations of the generators and Casimir operators of the related groups. Their transformation laws are obtained from pre-viously determined ones by the replacement of the parameters $j_{k}$ with the parameters $j_{k}^{\prime} j_{k}^{-1}$. We denote these transformations of generators and Casimir operators by $\Omega$. The inverse transformations $\Omega^{-1}$ are obtained from $\Omega$ by exchanging symbols with a prime for symbols without one and vice versa.

The map $\omega$ and the transformation $\Omega$ as well as $\omega^{-1}$ and $\Omega^{-1}$ are not well defined for all Cayley-Klein geometries and groups, considering that the symbols $\iota_{k}^{-1}, \iota_{m} \iota_{k}^{-1}$ for $k \neq m$ are not defined. We define only $\iota_{k} \iota_{k}^{-1}=1$ for $k=l, 2, \ldots, n$. Therefore, in the case where some parameter $j_{m}$ is equal to the dual unit, $j_{m}=\iota_{m}$, the maps (38) are valid when and only when a primed parameter with the same index is equal to the same dual unit, i.e., $j_{m}^{\prime}=\iota_{m}$. A simple analysis of the map (38) and the inverse map

$$
\begin{gathered}
\omega^{-1}: R_{n+1}\left(\mathbf{j}^{\prime}\right) \rightarrow R_{n+1}(\mathbf{j}) \\
\omega^{-1} x_{0}^{\prime}=x_{0}, \quad \omega^{-1} x_{k}=x_{k}^{\prime} \prod_{m=1}^{k} j_{m} j_{m}^{\prime-1}, \quad k=1,2, \ldots, n
\end{gathered}
$$

from the viewpoint of the validity of the transformations gives the following classification theorem (Gromov, 1982) (the same results hold for $\Omega$ and $\Omega^{-1}$ as far as they include the same combinations of parameters $j_{k}^{\prime} j_{k}^{-1}, j_{k} j_{k}^{\prime-1}$ as $\omega$ and $\left.\omega^{-1}\right)$.

Classification Theorem. 1. Let $S O_{n+1}(\mathbf{j})$ be the motion group of the Cayley-Klein space $R_{n+1}(\mathbf{j})$ with $j_{1} \neq \iota_{1}, \ldots$, and $j_{n} \neq \iota_{n}$; and let $S O_{n+1}\left(\mathbf{j}^{\prime}\right)$ be the motion group of the arbitrary Cayley-Klein space $R_{n+1}\left(\mathbf{j}^{\prime}\right)$; then $R_{n+1}\left(\mathbf{j}^{\prime}\right)=\omega R_{n+1}(\mathbf{j})$ and $S O_{n+1}\left(\mathbf{j}^{\prime}\right)=\Omega S O_{n+1}(\mathbf{j})$. If $j_{1}^{\prime} \neq \iota_{1}, \ldots$, and $j_{n}^{\prime} \neq$ $\iota_{n}$, then $\omega$ and $\Omega$ are one-to-one mappings and $R_{n+1}(\mathbf{j})=\omega^{-1} R_{n+1}\left(\mathbf{j}^{\prime}\right), S O_{n+1}(\mathbf{j})=$ $\Omega^{-1} S O_{n+1}\left(\mathbf{j}^{\prime}\right)$.

2. Let $S O_{n+1}(\mathbf{j})$ be the motion group of the Cayley-Klein space $R_{n+1}(\mathbf{j})$ with $j_{k_{1}}=\iota_{k_{1}}, \ldots, j_{k_{s}}=\iota_{k_{s}}$ and $S O_{n+1}\left(\mathbf{j}^{\prime}\right)$ be the motion group of the space $R_{n+1}\left(\mathbf{j}^{\prime}\right)$ with $j_{r_{1}}^{\prime}=\iota_{r_{1}}, \ldots, j_{r_{m}}^{\prime}=\iota_{r_{m}}, m>s$; then $R_{n+1}\left(\mathbf{j}^{\prime}\right)=$ $\omega R_{n+1}(\mathbf{j}), S O_{n+1}\left(\mathbf{j}^{\prime}\right)=\Omega S O_{n+1}(\mathbf{j})$ on the condition that the number set $\left(r_{1}, r_{2}, \ldots, r_{m}\right)$ contains the number set $\left(k_{1}, k_{2}, \ldots, k_{s}\right)$. The transformations $\omega$ and $\Omega$ are one-to-one if and only if $s=m ; k_{1}=r_{1}, \ldots, k_{s}=r_{m}$. In this case $R_{n+1}(\mathbf{j})=\omega^{-1} R_{n+1}\left(\mathbf{j}^{\prime}\right)$ and $S O_{n+1}(\mathbf{j})=\Omega^{-1} S O_{n+1}\left(\mathbf{j}^{\prime}\right)$.

From this theorem we have the following results.

Corollary. The group $S O_{n+1}(\mathbf{j})$ for any parameters $\mathbf{j}$ may be obtained from any group $S O_{n+1}(\mathbf{j})$ the parameters of which are not dual.

Of course the simplest groups are the classical ones and we began in the previous sections from these groups.

The contraction scheme for the kinematic groups (Bacry and LevyLeblond, 1968; Sanjuan, 1984) easily follows from the classification theorem. Indeed, according to the corollary, the de Sitter group $S_{5}(i, i, 1,1)$ contracts into the Poincare $S_{5}\left(\iota_{1}, i, 1,1\right)$ or the Galilei $S O_{5}\left(\iota_{1}, \iota_{2}, 1,1\right)$ groups or the Newton-Hooke group $S_{5}\left(i, \iota_{2}, 1,1\right)$. The Newton-Hooke group $S O_{5}\left(1, \iota_{2}, 1,1\right)$ 
or $\mathrm{SO}_{5}\left(i, \iota_{2}, 1,1\right)$ according to the second part of theorem contracts only into the Galilei group. The transitions from de Sitter group to the Carroll groups $S O_{5}\left(j_{1}, 1,1, \iota_{4}\right)$ are made with the help of contractions and analytical continuations.

\section{Conclusions}

Starting from Klein's fundamental idea that each geometry is defined by its motion group and using as a mathematical tool the unified description of all Cayley-Klein geometries, we have developed a simple but effective method of transitions between groups that gives the opportunity to obtain the generators, commutators, and Casimir operators of the motion group of an arbitrary Cayley-KJein space from those of the classical orthogonal group. Inonu-Wigner contractions and analytical continuations of the groups are naturally unified in the developed approach. The common nature of both processes was displayed, namely the continuation of the real group parameters into the dual or complex number fields. The structure of transitions between the Cayley-Klein groups is described by a classification theorem. Only contractions restrict the possible transitions between the groups. Physically important kinematic groups naturally arise in our approach. If one accepts that a physical theory is based on the group of the invariants of the physical phenomena described by this theory, then the method of the transitions provides a tool for the simultaneous investigation of one or another set of physical theories connected with each other by transitions. The special theory of relativity and classical mechanics, and field theory and statistical mechanics are well-known examples of such sets of theories.

\section{References}

[1] Bacry, H., and Levy-Leblond, J. M. (1968). Journal of Mathematical Physics, 9, 1605-1614.

[2] Barut, A. O., and Raczka, R. (1977). Theory of Group Representations and Applications, PWN-Polish Scientific Publishers, Warsaw.

[3] Bourbaki, N. (1967). Varietes Differentiates et Analytiques, Hermann, Paris.

[4] Chaichian, M., Demichev, A. P., and Nelipa, N. F. (1983). Communications in Mathematical Physics, 90, 353-372.

[5] Derom, J.-R., and Dubois, J.-G. (1972). Nuovo Cimento, 9B, 351-376.

[6] Fernandez Sanjuan, M. A. (1984). International Journal of Theoretical Physics, 23, 1-14.

[7] Gel'fand, I. M. (1950). Matematicheski Sbornik, 26, 103-112 (in Russian).

[8] Gromov, N. A. (1978). Preprint Series "Scientific Reports," No. 37, Komi Academy of Sciences, Syktyvkar, USSR (in Russian).

[9] Gromov, N. A. (1981). Teoreticheskaja i Matematicheskaja Fizika, 49, 210-218 (in Russian). 
[10] Gromov, N. A. (1982). Matematicheski Zametki, 32, 355-363 (in Russian).

[11] Gromov, N. A. (1984). Preprint Series "Scientific Reports," No. 95, Komi Academy of Sciences, Syktyvkar, USSR (in Russian).

[12] Gromov, N. A. (1985). Preprint Series "Scientific Reports," No. 126, Komi Academy of Sciences Syktyvkar, USSR (in Russian).

[13] Gromov, N. A. (1986). In Group Theoretical Methods in Physics, Nauka, Moscow, Vol. 2, pp. 183-198 (in Russian).

[14] Inonu, E., and Wigner, E. P. (1953). Proceedings of the National Academy of Sciences, USA, 39, 510-524.

[15] Levy-Leblond, J.-M. (1965). Annales de I'lnstitut Henri Poincare, A3, $1-12$.

[16] Levy-Leblond, J.-M. (1971). Group Theory and Its Applications, E. M. Loebl, eds., Academic Press, New York, Vol. 2, pp. 221-299.

[17] Lord, E. A. (1985). International Journal of Theoretical Physics, 24, 723-730.

[18] Mukunda, N. (1967). Journal of Mathematical Physics, 8, 2210-2220.

[19] Pimenov, R. I. (1965). Litovski Matematicheski Sbornik S, 457-486 (in Russian). 